\title{
Photothermal effects of laser-activated surface plasmonic gold nanoparticles on the apoptosis and osteogenesis of osteoblast-like cells
}

This article was published in the following Dove Press journal:

International Journal of Nanomedicine

27 July 2016

Number of times this article has been viewed

\author{
Lih-Rou Rau' \\ Wan-Yu Huang' \\ Jiunn-Woei Liaw ${ }^{2-5}$ \\ Shiao-Wen Tsai ${ }^{1,3,6}$ \\ 'Graduate Institute of Biochemical \\ and Biomedical Engineering, \\ ${ }^{2}$ Department of Mechanical \\ Engineering, ${ }^{3}$ Center for Biomedical \\ Engineering, Chang Gung University, \\ ${ }^{4}$ Institute for Radiological Research, \\ Chang Gung Memorial Hospital, \\ Chang Gung University, Taoyuan, \\ ${ }^{5}$ Center for Advanced Molecular \\ Imaging and Translation, ${ }^{6}$ Department \\ of Orthopaedic Surgery, Chang Gung \\ Memorial Hospital, Linkou, Taiwan, \\ Republic of China
}

\begin{abstract}
The specific properties of gold nanoparticles (AuNPs) make them a novel class of photothermal agents that can induce cancer cell damage and even death through the conversion of optical energy to thermal energy. Most relevant studies have focused on increasing the precision of cell targeting, improving the efficacy of energy transfer, and exploring additional functions. Nevertheless, most cells can uptake nanosized particles through nonspecific endocytosis; therefore, before hyperthermia via AuNPs can be applied for clinical use, it is important to understand the adverse optical-thermal effects of AuNPs on nontargeted cells. However, few studies have investigated the thermal effects induced by pulsed laser-activated AuNPs on nearby healthy cells due to nonspecific treatment. The aim of this study is to evaluate the photothermal effects induced by AuNPs plus a pulsed laser on MG63, an osteoblast-like cell line, specifically examining the effects on cell morphology, viability, death program, and differentiation. The cells were treated with media containing $50 \mathrm{~nm}$ AuNPs at a concentration of $5 \mathrm{ppm}$ for 1 hour. Cultured cells were then exposed to irradiation at $60 \mathrm{~mW} / \mathrm{cm}^{2}$ and $80 \mathrm{~mW} / \mathrm{cm}^{2}$ by a Nd:YAG laser (532 nm wavelength). We observed that the cytoskeletons of MG63 cells treated with bare AuNPs followed by pulsed laser irradiation were damaged, and these cells had few bubbles on the cell membrane compared with those that were not treated (control) or were treated with AuNPs or the laser alone. There were no significant differences between the AuNPs plus laser treatment group and the other groups in terms of cell viability, death program analysis results, or alkaline phosphatase and calcium accumulation during culture for up to 21 days. However, the calcium deposit areas in the cells treated with AuNPs plus laser were larger than those in other groups during the early culture period.

Keywords: pulsed laser, photothermal, gold nanoparticles, osteoblast, apoptosis, mineralization
\end{abstract}

\section{Introduction}

Hyperthermia, which increases cell membrane permeability and causes irreversible cell membrane damage to destroy cancer cells, is a noninvasive therapy approach. The heat generators commonly used in clinical practice include radiofrequency waves, ${ }^{1}$ microwaves, ${ }^{2}$ and ultrasound. ${ }^{3}$ However, the traditional heating sources for hyperthermia are limited because they can all easily damage surrounding healthy tissues. The identification of a method that restricts treatment to the pathological region, prevents harm to normal regions, and increases the efficacy is an underdeveloped issue in the field of cancer therapy. It is well known that cell viability decreases as laser power/ energy increases; therefore, development of a method that can efficiently reduce the laser energy necessary to destroy tumor cells is critical.

Localized surface plasmon resonance (LSPR) is a unique optical property of nanosized metallic particles that allows collective oscillation of free electrons in 
conduction bands exposed to an electromagnetic field. ${ }^{4}$ Laser excitation of the LSPR of gold nanoparticles (AuNPs) can induce photon-electron and electron-electron interactions and then generate heat. Tunable AuNPs could be used for various applications, such as photosensitive agents for hyperthermia therapy, ${ }^{5-7}$ triggers for thermally induced reactions, ${ }^{8}$ and biomarkers for cell labeling. ${ }^{9-11}$ Although the LSPR absorption peak of Au/AuNPs is size and geometry dependent, ${ }^{12,13}$ the critical parameter for the efficacy of AuNP-mediated laser-activated hyperthermia is the amount of energy delivered by the laser per time and area. The heat generated by plasmonic AuNPs is influenced by the following: the concentration of AuNPs; the wavelength of the laser, which is dependent on the LSPR absorption peak; and the type of laser used. In general, a pulsed laser delivers more energy than a continuous wave laser in a very short time. Therefore, it is hard to determine the optimal conditions by comparing previous studies (Table 1). Most studies agree that the photothermal ablation effect is more prominent with higher energy laser irradiation. However, high laser energy increases nonspecific cell death. Thus, as with conventional methods, AuNPs could also induce unavoidable cell damage in surrounding healthy tissues when used for laser-activated hyperthermia in cancer treatment.

To date, most studies have focused on increasing the efficacy of AuNP-induced hyperthermia to initiate cancer cell necrosis or inhibit growth. ${ }^{14-18}$ However, the yield of photothermal transformation by AuNPs is related to the amount taken up by cells and the laser intensity. ${ }^{19}$ Furthermore, the amount of AuNPs taken up by cells depends on several parameters, such as particle size, shape, surface charge, surface modification, and cell type. ${ }^{20-23}$ Unfortunately, there is no consensus on the laser parameters (eg, laser energy, intensity, and duration) that are needed to induce cell death through laser-activated photothermal effects (Table 1). In addition, most cells can uptake nanosized particles through nonspecific endocytosis; therefore, before hyperthermia via AuNPs can be applied for clinical use, it is important to understand the adverse optical-thermal effects of AuNPs on nontargeted cells.

To date, few studies have evaluated AuNP-mediated photothermal effects on the proliferation and differentiation of normal cells after laser exposure. In this work, we investigated the relative high- and low-power laser-induced photothermal effects on MG63 cells. The morphology, viability, and differentiation levels of MG63 cells treated with 5 ppm bare AuNPs after exposure to various laser intensities were compared to those of cells that were not irradiated and not treated with AuNPs.

\section{Materials and methods Cell culture}

In this study, MG63 osteoblast-like cells (human osteogenic sarcoma, BCRC number: 60279) were used to investigate the photothermal effects of laser-induced AuNPs on cellular behaviors. MG63 cells represent an immature osteoblast phenotype that can differentiate under induction culture conditions. ${ }^{24}$ Therefore, MG63 cells should express specific bone matrix mineralization-related markers, such as alkaline phosphatase (ALP), and also deposit calcium after culture in an induction culture medium. The induction medium was modified Eagle's medium, which contained 10\% fetal bovine serum and $1 \%$ antimicrobial agent supplemented with $50 \mu \mathrm{g} / \mathrm{mL}$ ascorbic acid and $10 \mathrm{mM}$ glycerol 2-phosphate disodium salt hydrate. The cells were incubated at $37^{\circ} \mathrm{C}$ in a humidified atmosphere of $5 \% \mathrm{CO}_{2}$, and the culture medium was changed once every 3 days.

\section{Synthesis of AuNPs}

AuNPs were synthesized via the citrate reduction of a gold salt. Briefly, $200 \mathrm{~mL}$ of deionized water containing $100 \mu \mathrm{L}$ of $0.5 \mathrm{M} \mathrm{HAuCl}_{4}$ and $100 \mu \mathrm{L}$ of $1 \%$ sodium citrate were heated to boiling for 15 minutes. The other chemicals were of reagent grade and were purchased from Sigma-Aldrich Co. (St Louis, MO, USA). The sizes of the nanoparticles were measured by randomly choosing 100 AuNPs from transmission electron

Table I Comparison of the results from previous studies on regarding photothermal effects on cell viability

\begin{tabular}{|c|c|c|c|c|c|}
\hline \multicolumn{2}{|l|}{ AuNPs } & \multicolumn{2}{|l|}{ Laser } & \multirow[t]{2}{*}{ Cell viability } & \multirow[t]{2}{*}{ References } \\
\hline Size, $\mathbf{n m}$ & Concentration & Type/wavelength, nm & Power; duration & & \\
\hline 15 & $10^{4} / \mathrm{mL}$ & Pulsed laser/532 & $48 \mathrm{~J} / 5$ pulse & $\sim 75 \%$ & 15 \\
\hline 60 & $0.2 \mathrm{nM}$ & Diode laser/980 & $2,700 \mathrm{~J} / \mathrm{cm}^{2} ; 3 \mathrm{~min}$ & $\sim 0 \%$ & 17 \\
\hline 30 & $7.3 \times 10^{10} / \mathrm{mL}$ & Diode laser/808 & $2,400 \mathrm{~J} / \mathrm{cm}^{2} ; 5 \mathrm{~min}$ & $\sim 100 \%$ & 18 \\
\hline 100 & $8.3 \mu \mathrm{g} / \mathrm{mL}$ & Pulsed laser $/ 532$ & $25-50 \mathrm{~mJ} / \mathrm{cm}^{2} ; 90 \mathrm{~s}$ & $\sim 0 \%\left(>9 \mathrm{~W} / \mathrm{cm}^{2}\right)$ & 19 \\
\hline 15 & $6.03 \times 10^{15} / \mathrm{mL}$ & Diode laser/540 & $6,000 \mathrm{~J} / \mathrm{cm}^{2} ; 5 \mathrm{~min}$ & $\sim 0 \%$ & 28 \\
\hline 15 & $2 \mathrm{nM}$ & Argon laser $/ 514$ & $6,000 \mathrm{~J} / \mathrm{cm}^{2} ; 2 \mathrm{~min}$ & $\sim 0 \%$ & 29 \\
\hline
\end{tabular}

Note: Laser power was converted to the same unit.

Abbreviations: AuNP, gold nanoparticle; min, minutes; s, seconds. 
microscopic (JEOL 1230) images. The concentrations of AuNPs in the media were measured using inductively coupled plasma atomic emission spectroscopy. ${ }^{9,25}$

\section{Laser set-up}

We previously set up a laser-induced microbubble configuration to investigate the Faraday-Tyndall effect of AuNPs on the laser-induced microbubble, ${ }^{26}$ and this equipment was used to evaluate the laser-activated photothermal effects of AuNPs on living cells. Briefly, an optical parametric oscillator attached to a Q-switched Nd:YAG laser (532 nm wavelength) with a second harmonic generator (SureliteII-10; Continuum, San Jose, CA, USA) was used with a spot size diameter of $10 \mathrm{~mm}$. The emitted laser light had a repetition rate of $10 \mathrm{~Hz}$, and the exposure times were fixed to 1 minute for all experiments. The pulsed laser output power was measured by a handheld optical power meter. The laser power was varied using an attenuator placed between the laser and the first mirror to yield cell irradiances of $60 \mathrm{~mW} / \mathrm{cm}^{2}$ and $80 \mathrm{~mW} / \mathrm{cm}^{2}$. Then, homogenous laser irradiation was applied to a sample placed in a 24-well culture plate with a $1.5 \mathrm{~mm}$ diameter glass slide.

\section{Determining the photothermal effects on cells}

To investigate the laser-activated photothermal effects of AuNPs on cellular behavior, six experimental groups were used: cells without any treatment (negative control group), cells treated with AuNPs alone, cells exposed to laser $\left(60 \mathrm{~mW} / \mathrm{cm}^{2}\right.$ or $\left.80 \mathrm{~mW} / \mathrm{cm}^{2}\right)$ alone, and cells treated with AuNPs and exposed to laser $\left(60 \mathrm{~mW} / \mathrm{cm}^{2}\right.$ or $\left.80 \mathrm{~mW} / \mathrm{cm}^{2}\right)$. The laser wavelength was $532 \mathrm{~nm}$, and the irradiation lasted for 1 minute at $60 \mathrm{~mW} / \mathrm{cm}^{2}$ or $80 \mathrm{~mW} / \mathrm{cm}^{2}$. After laser irradiation, we evaluated the cell morphology and the AuNP distribution inside the cells using dark-field microscopy. The dark-field hyperspectral imaging system (Cytoviva, Auburn, AL, USA) included an Olympus BX51 microscope (Olympus Corporation, Tokyo, Japan) and a high numerical dark-field condenser (UDCW, 1.2-1.4; Cytoviva). A 100× oil Iris objective was used, and the dark-field and bright-field images were captured with an Olympus DP72 single-chip color CCD camera (Olympus Corporation).

\section{Optical imaging of cells}

Cellular morphology was investigated by examining the staining of three cell components: the F-actin cytoskeleton, the nucleus, and the lipid molecules of membranes, which were stained with Texas Red-X phalloidin, 4',6-diamidino2-phenylindole (DAPI), and Annexin V-Alexa Fluor 488 (Thermo Fisher Scientific, Waltham, MA, USA), respectively.
After laser irradiation, Annexin V-Alexa Fluor 488 was added to the cell media for 15 minutes; next, the cells were washed three times with phosphate-buffered saline (PBS), then fixed in a $4 \%$ paraformaldehyde solution for 15 minutes at room temperature, and finally treated with $0.1 \%$ Triton X-100 for 5 minutes. To reduce nonspecific background staining, the samples were blocked with $1 \%$ bovine serum albumin in PBS for 30 minutes. After the bovine serum albumin solution was aspirated, the samples were incubated with $200 \mu \mathrm{L}$ of Texas Red-X phalloidin for 20 minutes. The samples were then incubated with DAPI solution for 15 minutes to stain the DNA in the nuclei of the cells. After three 5-minute washes with PBS, the samples were observed under a laser scanning confocal microscope (Zeiss LSM 510 META). The light scattered from AuNPs is monochromatic, and its wavelength is the same as that of the laser.

\section{Determination of cell viability}

The water-soluble tetrazolium (WST assay used to determine cell viability is based on the reductive cleavage of tetrazolium salt (WST-1) to soluble formazan by mitochondrial dehydrogenase, which is only active in viable cells. The pretreated cells were collected at various time intervals after inoculation. The WST reagent was added, and the samples were incubated further at $37^{\circ} \mathrm{C}$ and $5 \% \mathrm{CO}_{2}$ for 3 hours. Experiments were performed in triplicate. The amount of dye formed was then immediately measured at a wavelength of $450 \mathrm{~nm}$.

\section{Alkaline phosphatase activity assay}

The ALP released in the medium after treatment was assayed according to the manufacturer's protocol (Sigma-Aldrich Co.). In brief, the pretreated cells were collected from the media at various time intervals after inoculation. After the cells were washed with PBS, lysate solution, which comprised $0.4 \mathrm{~mL}$ of PBS containing $0.1 \mathrm{M}$ glycine, $1 \mathrm{mM} \mathrm{MgCl}_{2}$, and $0.05 \%$ Triton X-100, was added, and the mixture was held at room temperature for 20 minutes. The sample was then incubated with $400 \mu \mathrm{L}$ of p-nitrophenyl phosphate solution at $37^{\circ} \mathrm{C}$ for 30 minutes. Enzyme activity was terminated by the addition of $200 \mu \mathrm{L}$ of ice-cold $3 \mathrm{~N} \mathrm{NaOH}$, and the amount of p-nitrophenol liberated was measured by monitoring the optical absorbance at $405 \mathrm{~nm}$. The amount of p-nitrophenol is considered as a measure of the ALP activity.

\section{Detection and quantification of mineralization}

Alizarin red-S staining was used to observe the formation of calcium phosphate by MG63 cells. ${ }^{27}$ Briefly, the postexposed cells were washed with PBS and then fixed in a 
$4 \%$ paraformaldehyde solution at room temperature for 15 minutes. The cell layers were then rinsed with water before being stained with $2 \%$ Alizarin red-S, $\mathrm{pH} 4.2$, for 15 minutes. After aspiration of the unbound dye, the stained cell layers were washed with deionized water and visualized under an inverted phase microscope. For quantification of mineralization, the bound stain was completely eluted by the addition of $800 \mu \mathrm{L}$ of $10 \%$ acetic acid to each well at room temperature for 30 minutes to detach the cell layer from the glass slide. Then, cells were scraped and transferred to a microcentrifuge tube with $10 \%$ (v/v) acetic acid. After a short period of vortexing, which heated the mixture to $85^{\circ} \mathrm{C}$ for 10 minutes, the mixture was transferred to ice and allowed to cool to room temperature. The mixture was then centrifuged at $20,000 \times g$ for 15 minutes and mixed well with $500 \mu \mathrm{L}$ of the supernatant and $200 \mu \mathrm{L}$ of $10 \%(\mathrm{v} / \mathrm{v})$ ammonium hydroxide to neutralize the acid. The absorbance of the supernatant was measured at $405 \mathrm{~nm}$.

\section{Statistical analyses}

The experiments were conducted in triplicate, and the results were expressed as the mean $\pm \mathrm{SD}$. Statistical analyses were performed using the SPSS v.10 (IBM Corporation, Armonk, NY, USA) software package. Cellular viability and ALP activity were analyzed using the nonparametric KruskalWallis $H$-test, and if significance was found at $P<0.05$, the individual Mann-Whitney test was conducted to determine the differences between groups. Differences of $P<0.05$ were considered statistically significant.

\section{Results}

\section{Photothermal effects on cellular morphology}

The synthesis and characterization methods of AuNPs such as transmission electron microscopy-energy dispersive spectroscopy and Fourier transform infrared spectroscopy have been published in our previous studies. ${ }^{9,25}$ The average size of the AuNPs used in this work was $50.88 \pm 7.56 \mathrm{~nm}$, which was determined by examining 100 randomly selected particles in transmission electron microscopic images. The ultraviolet-visible (UV-vis) spectrum showed that the major surface plasmonic resonance adsorption peak was $533 \mathrm{~nm}$ (Figure S1). Therefore, we chose a Nd:YAG-pulsed laser with $532 \mathrm{~nm}$ as the light source for investigating the AuNPmediated photothermal effects on cellular behavior. As shown in Figure 1, AuNP treatment or laser irradiation alone did not alter the morphology of MG63 cells compared with untreated cells; however, some microbubbles were found on the surface of cells containing AuNPs after laser exposure. Additionally, the number of microbubbles increased as the laser power increased (Figure $1 \mathrm{E}$ and $\mathrm{F}$ ).

Annexin V-Alexa Fluor 488 has often been used to characterize the integrity of the cellular membrane. In general, the phosphatidylserine lipid molecules of membranes are located in the intracellular plasma membrane and thus cannot bind to Annexin V. However, once the membrane begins to break down, phosphatidylserine is externalized to the extracellular plasma membrane and can be tracked using Annexin V (Figure S2). In addition, the cell nuclei were stained with DAPI, and the cytoskeletons were stained with Texas Red-X phalloidin to enable observation of photothermal-induced cellular morphology changes by dark-field microscopy and fluorescence microscopy. Regardless of the experimental group, the cell membrane maintained its integrity, which was observed by the absence of Annexin V-stained images (Figure 2A). These photographs indicated that treatment with AuNPs plus laser irradiation, AuNPs alone, and laser alone did not disrupt the cellular membrane. However, the postexposure elongation degree of cells containing AuNPs
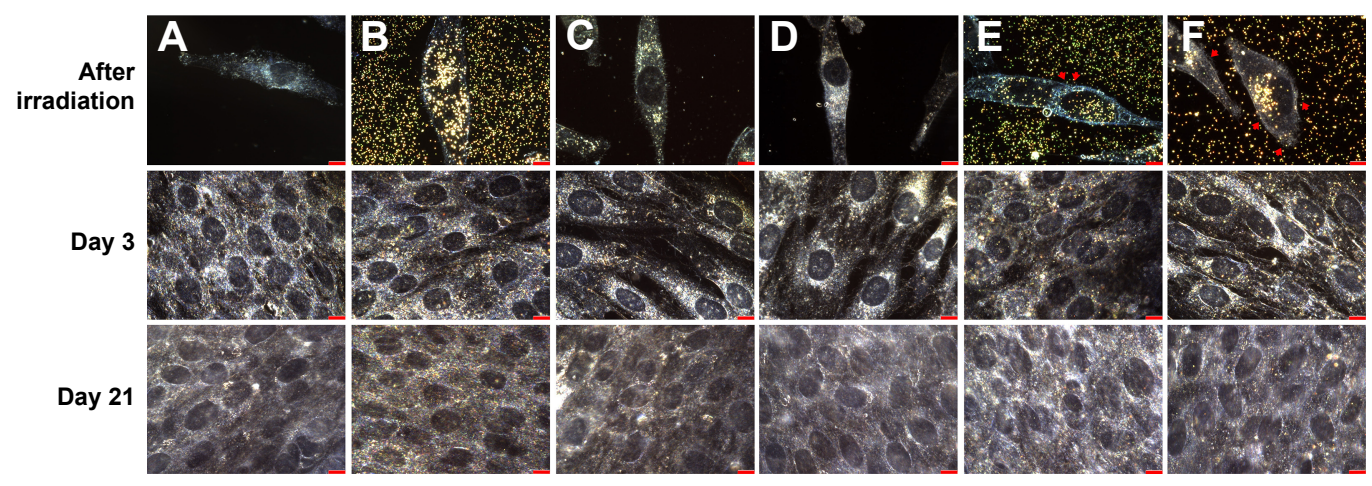

Figure I Dark-field image of cells.

Notes: (A) Untreated (negative control), (B) AuNPs alone, (C) after $60 \mathrm{~mW} / \mathrm{cm}^{2}$ laser exposure for I minute alone, (D) after $80 \mathrm{~mW} / \mathrm{cm}^{2}$ laser exposure for I minute alone, (E) AuNPs plus $60 \mathrm{~mW} / \mathrm{cm}^{2}$ laser for I minute, and (F) AuNPs plus $80 \mathrm{~mW} / \mathrm{cm}^{2}$ laser for I minute. Arrow head indicates bubbles present on the cells treated with AuNPs plus laser. Scale bar: $10 \mu \mathrm{m}$. Images were taken with a dark-field microscope at $100 \times$ magnification.

Abbreviation: AuNP, gold nanoparticle. 


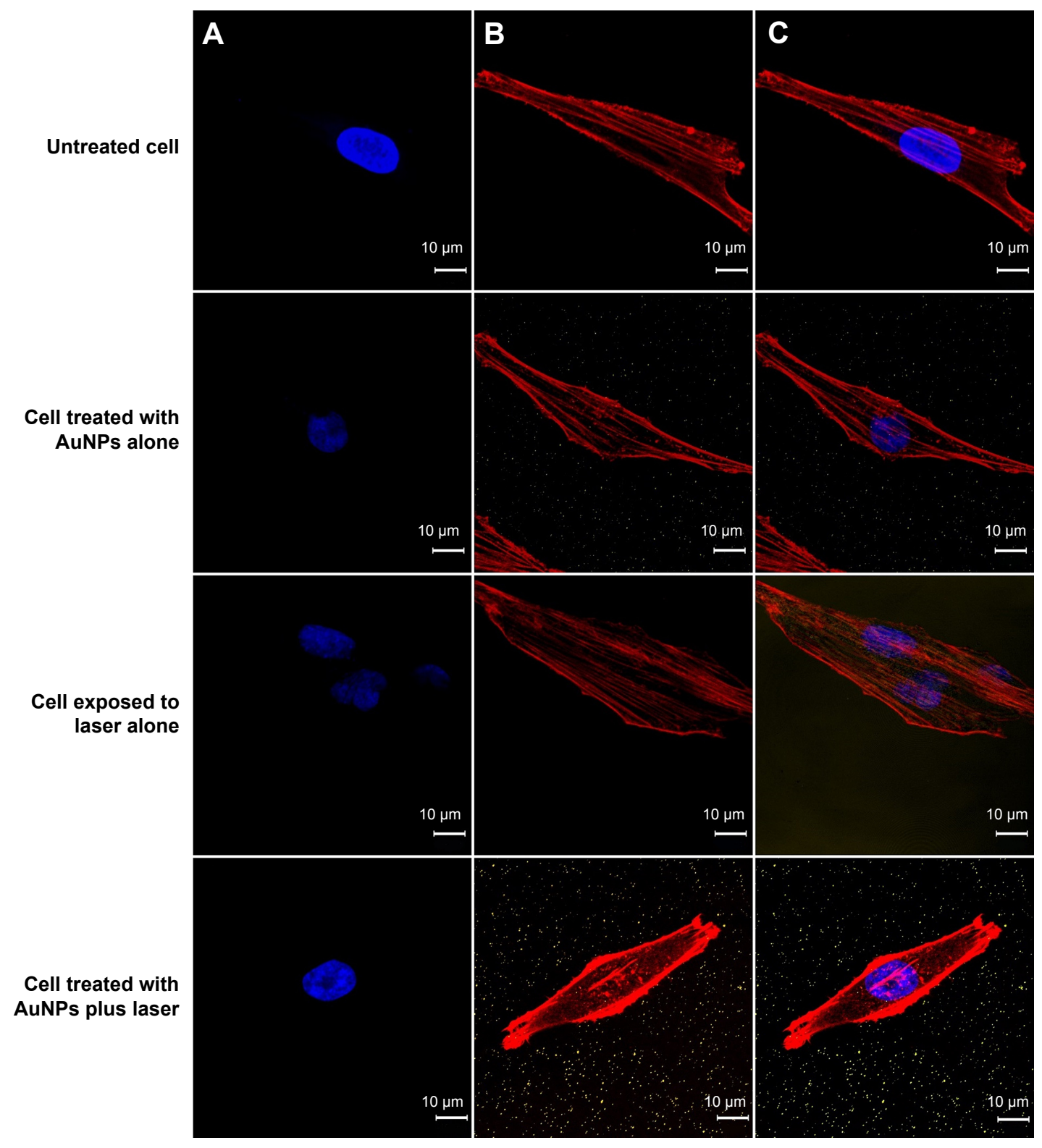

Figure 2 Confocal microscopy photograph of cells.

Notes: Experimental groups were untreated (negative control), AuNPs alone, $80 \mathrm{~mW} / \mathrm{cm}^{2}$ laser exposure for I minute alone, and AuNPs plus laser exposure for I minute. (A) Annexin V-Alexa Fluor 488 was used to indicate PS, shown in green; DAPI was used to stain nuclei, shown in blue; (B) Texas Red-X phalloidin was used to label cytoskeletal F-actin, shown in red; and (C) superimposition of $(\mathbf{A})$ and $(\mathbf{B})$. No green spots were observed in any of the groups. Images were taken at I00 $\times$ magnification. Abbreviations: AuNP, gold nanoparticle; PS, phosphatidylserine; DAPI, 4',6-diamidino-2-phenylindole.

was less than that of cells without AuNPs (Figures 1F and 2). Notably, the F-actin filaments were fractured into several short segments when cells were treated with AuNPs and irradiated at $80 \mathrm{~mW} / \mathrm{cm}^{2}$ (Figure $2 \mathrm{~B}$ ). In contrast, cells without AuNPs under the same laser irradiation conditions showed no difference from the control group. Although the cells containing AuNPs appeared to be damaged by laser irradiation, they recovered for further culture. As shown in Figure 1, there is no difference in the cell morphology, including shape and extension degree, for all groups after a fresh culture of up to 21 days.

\section{Photothermal effects on viability and the cell death program}

The recovery culture was initiated immediately after irradiation and was WST assayed on days 1, 3, 7, 14, and 21; the results showed that all groups presented similar growth rates without significant differences for 21 days (Figure 3, $P>0.05$ ). In addition, to understand the photothermal effects of postirradiated cells with fragmented actin filaments on the cellular death pathway, we analyzed cell apoptosis and necrosis percentages using Annexin $V$ and propidium iodide double staining. The results are shown in Figure 4, the four-quadrant 


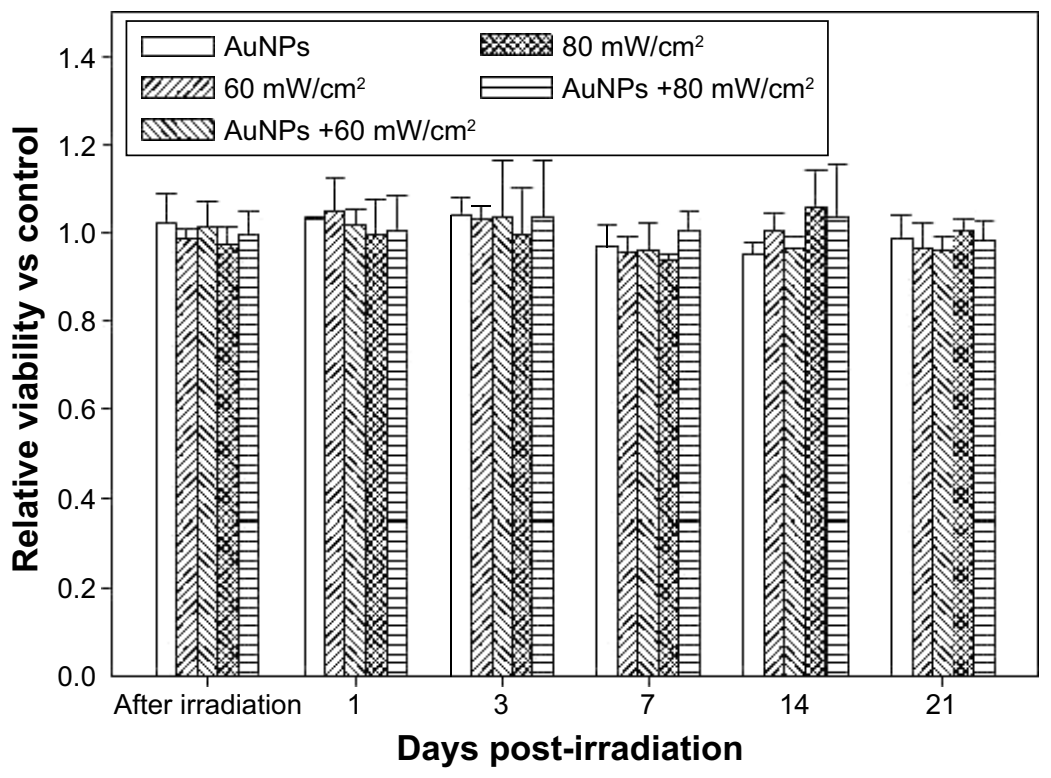

Figure 3 Photothermal effects on the viability of MG63 cells.

Notes: The viability of MG63 cells treated with $5 \mathrm{ppm}$ AuNPs and laser irradiation of either $60 \mathrm{~mW} / \mathrm{cm}^{2}$ or $80 \mathrm{~mW} / \mathrm{cm}^{2}$ for I minute and then cultured in fresh medium for 21 days. Data are presented as the mean $\pm S D(n=9)$ and were analyzed using the nonparametric Kruskal-Wallis $H$-test. Differences of $P<0.05$ were considered statistically significant.

Abbreviations: AuNP, gold nanoparticle; SD, standard deviation.

plots in each panel show the necrotic cells (upper left), the viable cells (lower left), the early apoptosis cells (lower right), and the late apoptosis cells (upper right). The four-quadrant plots for all of the experimental groups indicate similar findings compared with untreated cells (control group). Furthermore, we used the flow cytometer associated with the Cell Quest software package (Becton, Dickinson and Company, Franklin Lakes, NJ, USA) to quantify the distribution of cells within the cell death program. As shown in Figure 4E, there are no obvious differences in the percentages of necrotic, viable, early apoptosis, and late apoptosis cells among all of the treated cell groups and the control group. These results indicate that the laser-activated AuNP-mediated photothermal effects do not alter the cell death program and do not affect cell viability (Figure 4E; $P>0.05$ ).

\section{Photothermal effects on ALP expression and mineralization}

The phenotypic characteristics of MG63 cells were evaluated using ALP activity, which is an essential early marker of osteoblast differentiation. The expression levels of cells treated with AuNPs plus laser after culturing for up to 21 days are shown in Figure 5. There were no significant differences among all groups, including the control group, on each evaluated day. The results indicate that the photothermal effects activated by AuNPs plus laser in the study did not alter the progress of the early differentiation of osteoblasts. The colorimetric calcium quantification of Alizarin red-S staining was used to evaluate calcium deposition, which is considered an important late function of osteoblasts, on days 14 and 21 (Figure 6A and B). The results clearly show that the mineralization was time dependent. There was no significant difference in total calcium deposition among all groups (Figure 6C). However, the size of Alizarin red-S staining spots in laser-exposed cells containing AuNPs on day 14 and day 21 was significantly larger than those in the untreated, laser exposure-only, and AuNP-only groups (Figure 6). Therefore, we further analyzed the colorimetric quantification by spot size, as shown in Figure 6D. The results illustrate that calcium deposition spots in the laser-activated AuNPmediated photothermal groups are significantly larger than in the control group in the early culture period. The results indicated that although the total calcium deposition does not differ between any of the experimental groups after culturing for up to 21 days, the laser-activated AuNP-mediated photothermal effects actually promote calcium deposition by osteoblasts compared with the control group.

\section{Discussion}

Hyperthermia due to laser-activated AuNPs represents a noninvasive method of local overheating that can efficiently target cancer..$^{5-7}$ Raji et al ${ }^{28}$ demonstrated that AuNPs conjugated to epidermal growth factor receptor antibodies could selectively target human epithelial cancer cells (A431) and induce cell death after laser irradiation. $\mathrm{Li}$ and $\mathrm{Gu}^{14}$ reported that irradiating cancer cells with a high-power laser 

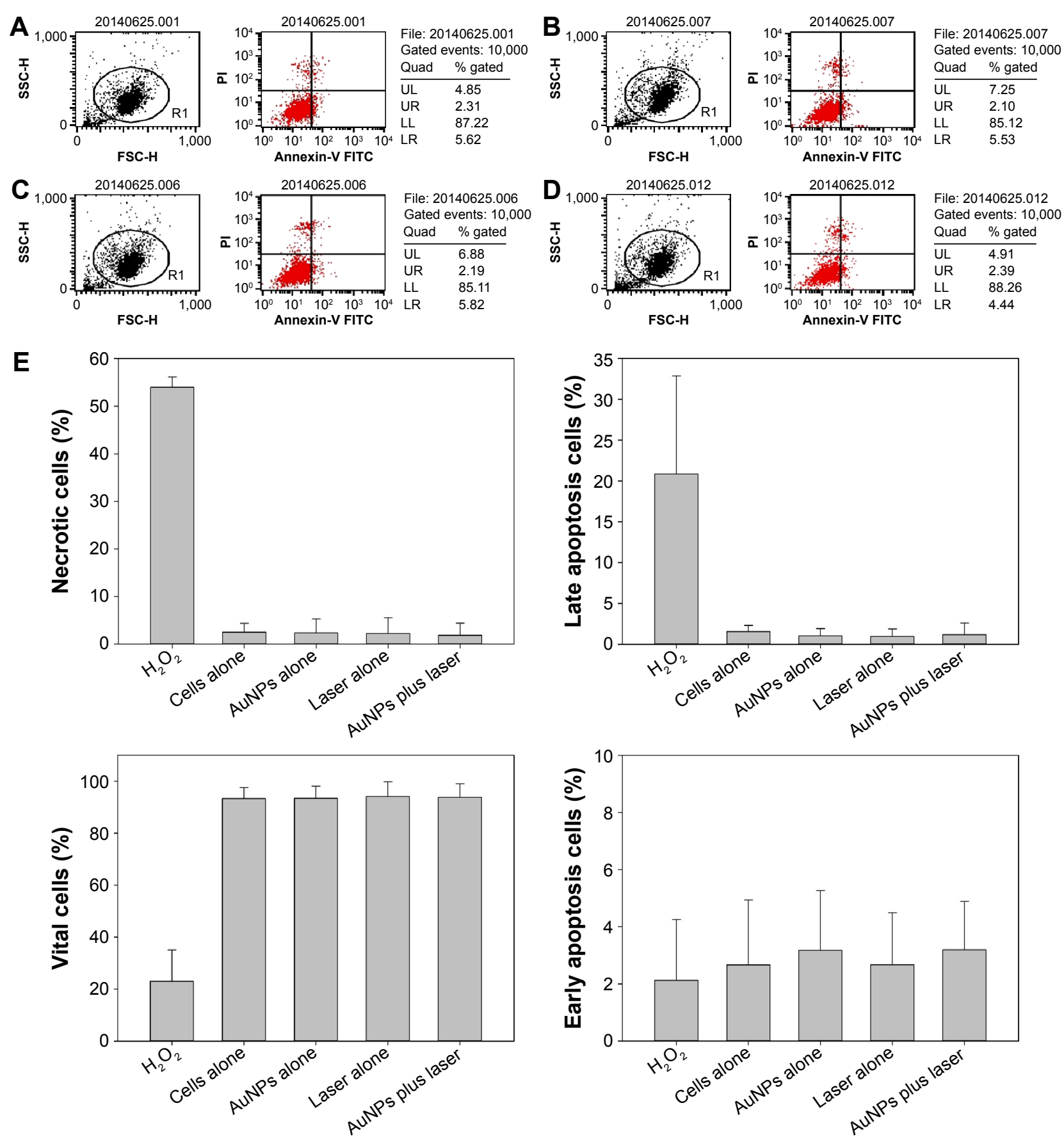

Figure 4 Photothermal effects on the cell death program of MG63 cells.

Notes: Cells treated with 5 ppm of AuNPs and laser irradiation of $80 \mathrm{~mW} / \mathrm{cm}^{2}$ for I minute and then cultured in fresh medium for I day. (A) Cells alone, (B) AuNPs alone, (C) laser alone, and (D) AuNPs plus laser. Dot plots of Annexin V/PI staining are shown. The upper-left quadrant shows the necrotic (Annexin V-/PI+) population. The upper-right quadrant shows the late apoptotic/necrotic (Annexin $\mathrm{V}+/ \mathrm{PI}+$ ) population. The lower-left quadrant shows the vital (Annexin $\mathrm{V}-/ \mathrm{PI}-$ ) population. The lower-right quadrant shows the early apoptosis (Annexin $\mathrm{V}+/ \mathrm{PI}-$ ) population. The results are from one experiment and are representative of three similar independent experiments.

(E) The percentages of viable cells, early apoptosis cells, late apoptosis cells, and necrotic cells are summarized from three independent experiments and are presented as the mean \pm SD. Data were analyzed using the nonparametric Kruskal-Wallis $H$-test. Differences of $P<0.05$ were considered statistically significant.

Abbreviations: AuNP, gold nanoparticle; PI, propidium iodide; UL, upper left; UR, upper right; LL, lower left; LR, lower right; FITC, fluorescein isothiocyanate; SD, standard deviation; FSC-H, forward scatter-height of intensity; SSC-H, side scatter-height of intensity.

definitively induced cell necrosis, but the process also had the possibility of damaging nearby normal cells. However, the photothermal properties of AuNPs can be used to decrease the necessary laser power and thus reduce the side effects on normal cells and increase the apoptosis of cancer cells.
Similar results were reported by $\mathrm{Qu}$ et al ${ }^{15}$ who evaluated BerH2-AuNPs (containing an antibody against CD30 receptors) and ACT1-AuNPs (containing an antibody against CD25 receptors) and found that laser irradiation induced the cell death of L428 cells, which have CD30 receptors. 


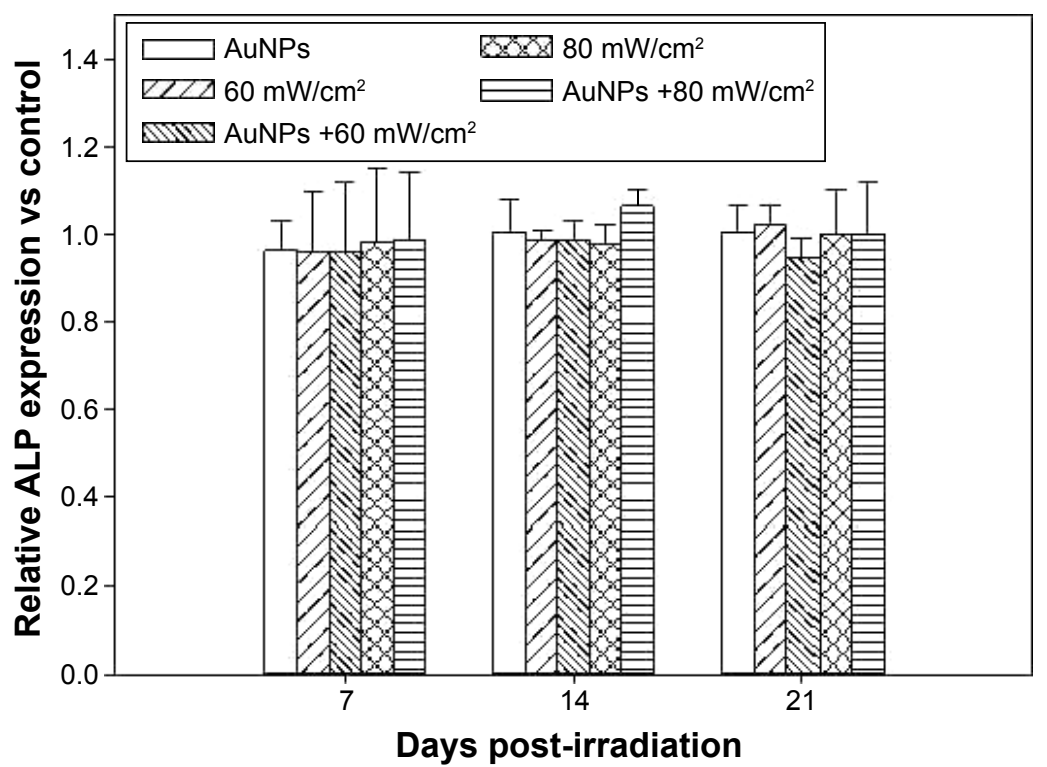

Figure 5 Photothermal effects on the ALP expression of MG63 cells.

Notes: The MG63 cells were treated with $5 \mathrm{ppm}$ of AuNPs and laser irradiation of either $60 \mathrm{~mW} / \mathrm{cm}^{2}$ or $80 \mathrm{~mW} / \mathrm{cm}^{2}$ for I minute and then cultured in fresh medium for $2 \mathrm{I}$ days. Data are presented as the mean $\pm S D(n=9)$ and were analyzed using the nonparametric Kruskal-Wallis $H$-test. Differences of $P<0.05$ were considered statistically significant. Abbreviations: AuNP, gold nanoparticle; ALP, alkaline phosphatase; SD, standard deviation.

A
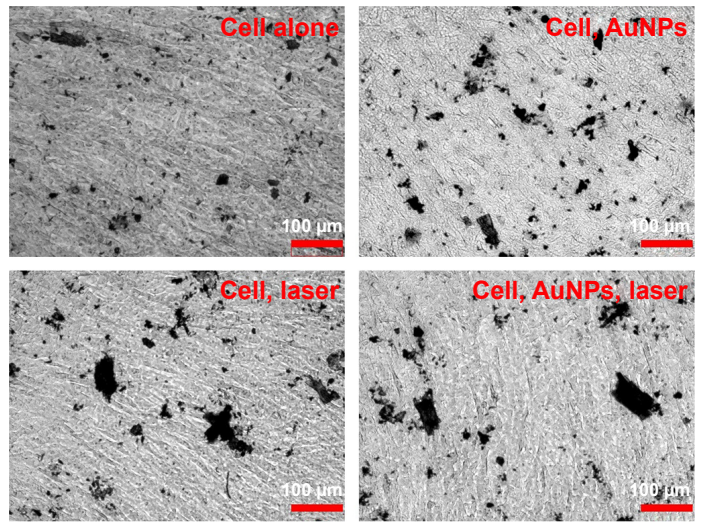

C

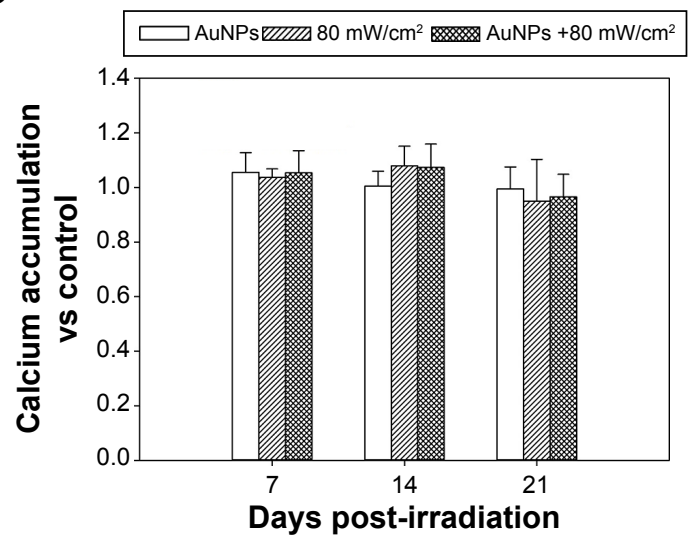

B
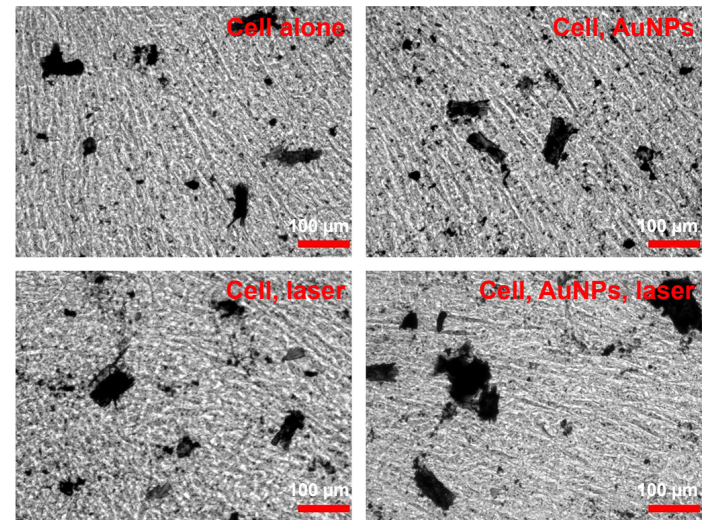

D
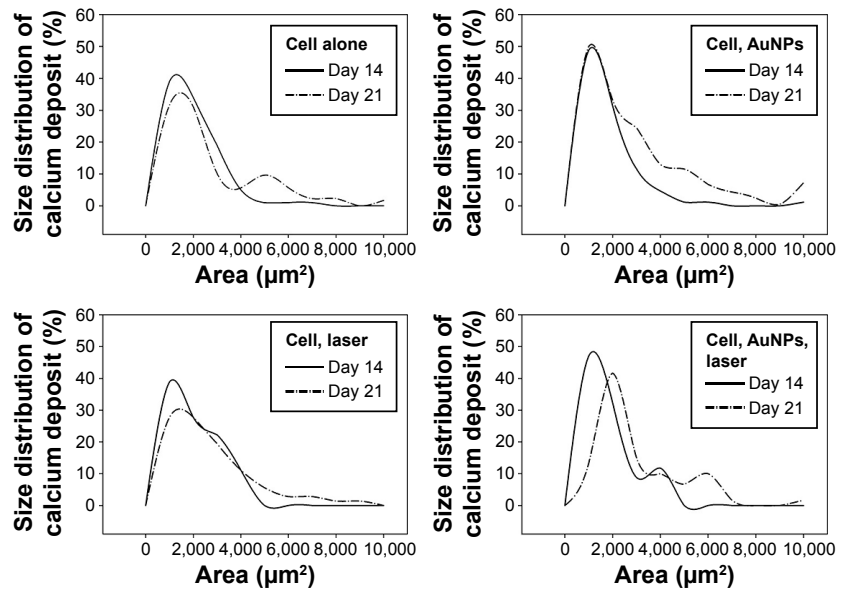

Figure 6 Photothermal effects on the calcium deposition of MG63 cells.

Notes: The mineralization activity of MG63 cells treated with $5 \mathrm{ppm}$ of AuNP and laser irradiation of $80 \mathrm{~mW} / \mathrm{cm}^{2}$ for I minute and then cultured in fresh medium for $2 \mathrm{I}$ days. The photographs of Alizarin red-S staining of mineralization in cells were taken on (A) day 14 and (B) day 21 . (C) Quantification of mineral deposition by Alizarin Red-S staining is presented as the mean $\pm S D, n=3$. Size distribution of mineral deposition was determined by examining 100 randomly selected AuNPs of Alizarin Red-S staining in photographs. The data are presented as the mean. (D) Data were analyzed using the nonparametric Kruskal-Wallis $H$-test. Differences of $P<0.05$ were considered statistically significant. Abbreviations: AuNP, gold nanoparticle; SD, standard deviation. 
In contrast, treatment with BerH2-AuNP initiated almost total cell death $(96.1 \%)$; this value was significantly higher than after treatment with ACT1-AuNPs (23.7\%). These results also showed that AuNPs can be taken up through nonselective ligand-receptor-medicated endocytosis; after laser irradiation, these AuNPs can then cause cell death. In addition, Krpetić et al ${ }^{29}$ demonstrated that photothermal transfer by laser-activated AuNPs led to cell necrosis if the AuNPs were taken up by receptor-mediated endocytosis to reach the cytoplasm. However, if the AuNPs did not undergo endocytosis by cells and were only located on the membrane, the photothermal effects only led to cell damage; the cells could recover after further culture. These previous studies indicated that even nontargeted cells are affected by laser exposure, such as membrane blebbing or a decreased metabolism rate; these effects depend on the laser intensity, duration, and amount of AuNP uptake. Nevertheless, the fraction of living cells can increase gradually after irradiation, indicating that the proliferation capacity was not altered by laser treatment.

Numerous studies have demonstrated that under irradiation, AuNPs can locally amplify the electromagnetic field and cause a cavitation bubble of submicron size. ${ }^{30-32}$ These studies inferred that cell death was caused by a lack of integrity in the cell membrane rather than the initiation of the cell death process. The results of this study are consistent with such conclusions. As shown in Figure 4, analysis of the cell death program found no significant difference between cells treated with AuNPs followed by pulsed laser irradiation and others groups (control, AuNPs alone, and irradiation alone). However, some bubbles were present on the cells treated with AuNPs plus laser, although no observable bubbles were found on other groups (Figure 1). A plausible suggestion for the mechanism underlying the disrupted cytoskeleton of cells treated with AuNPs plus laser may be the involvement of the bubbles. The bubbles generated by pulsed laser-activated AuNPs exist in the cytoplasm in a form similar to bombs, which cause a transient sufficiently high localized vapor pressure to disrupt the cytoskeleton. After treatment with AuNPs plus laser, the cytoskeleton was disrupted; thus, the cell extension was less than that of the control and other groups (Figure 2). Our previous work also demonstrated that the cell membrane will be lysed after exposure to a $532 \mathrm{~nm}$ pulsed laser at $100 \mathrm{~mW} / \mathrm{cm}^{2}$ for 1 minute (Figure S3). However, in the present work, the pulsed laser was used at relatively low power, so the total amount of heat generated cannot cause photothermal ablation of cells. As long as the mechanical damage was transient, the mitochondrial activity was not affected by the photothermal energy conversion, and the cells were recovered for further fresh culture and growth with the same trend as other groups.

Mvula et $\mathrm{al}^{33}$ exposed human adipose-derived stem cells to a $635 \mathrm{~nm}$ diode laser of $5 \mathrm{~J} / \mathrm{cm}$ and found there was no difference in cell morphology; instead, the cell viability increased 24 hours after treatment compared with that in a control group. Soleimani et $\mathrm{al}^{34}$ used a gallium aluminum arsenide laser on bone marrow mesenchymal stem cells and also observed that cells had higher ALP expression levels after laser irradiation. Yi et $\mathrm{al}^{35}$ and Sathuluri et $\mathrm{al}^{36}$ found that treating stem cells with AuNPs could enhance mineralization and proliferation, respectively. These studies indicate that AuNPs or a low-power laser can promote cell differentiation. In this study, we observed similar cell behavior. The ALP expression levels (Figure 5) and quantified calcium deposition (Figure 6D), however, showed no significant differences. Nevertheless, as shown in Figure 6E, the cells treated with AuNPs plus laser deposited larger areas of calcium than the other groups deposited. Together, all of the results indicate that AuNPs plus low energy laser may promote cell differentiation compared with control, AuNPs alone, and irradiation alone. However, the actual mechanisms by which lasers or AuNPs promote cell viability or differentiation are not very clear. One hypothesis suggests the involvement of free radicals and singlet oxygen in the process, but more precise experiments need to be designed and conducted to prove this hypothesis.

\section{Conclusion}

Given that AuNPs have high biocompatibility and low cytotoxicity, various biomedical applications have been conducted to determine the photothermal effects of laseractivated AuNPs. However, to date, most of the studies on hyperthermia-like effects have focused on how to kill cells more efficiently by continuous wave laser and how to treat deeper or larger areas of tissue. AuNPs can be well controlled in terms of their size and shape through fabrication parameters, and gold provides a flexible surface chemistry for conjugating molecules through specific bonds to specific cancer cells. Nevertheless, nontarget cells can take up AuNPs through nonspecific endocytosis. Therefore, it is very important to understand the photothermal effects exerted on nonspecific cells. In the present work, we used bare AuNPs, rather than surface-modified AuNPs, to investigate plasmonic photothermal effects on nontarget cells that can take up AuNPs through nonspecific endocytosis. Consideration of all findings together indicates that the photothermal effects induced by laser-activated AuNPs on nontarget cells were transient, with no influence on the cell maturation process. 
Moreover, a low-energy laser plus AuNPs may be capable of enhancing the early mineralization of MG63 cells. This result implies that treatment with AuNPs combined with a low-irradiance-pulsed laser may enhance the early mineralization of MG63 cells. This finding might pave the way for the application of laser-activated AuNPs on cellular differentiation. Meanwhile, AuNPs can reduce the laser energy that must be applied to damage tumor cells and cause nonvital damage of healthy cells, but the photothermal effects exerted by laser-activated AuNPs also have the potential to enhance stem cell differentiation. Therefore, in the future, it is important to understand what signal pathways trigger this response in order to control the yield of photothermal transformation mediated by AuNPs.

\section{Acknowledgments}

The authors are grateful to the Molecular Imaging Center at Chang Gung Memorial Hospital for providing hyperspectral microscopy. This research was supported by the National Science Council, Taiwan, Republic of China. (NSC 103-2221E-182-012) and by Chang Gung Memorial Hospital (CMRPD2A0052, CMRPD2A0053, and CIRPD2E0031).

\section{Disclosure}

The authors report no conflicts of interest in this work.

\section{References}

1. Paulides MM, Vossen SH, Zwamborn AP, Van Rhoon GC. Theoretical investigation into the feasibility to deposit RF energy centrally in the head-and-neck region. Int J Radiat Oncol Biol Phys. 2005;63(2): 634-642.

2. Dobsícek Trefná H, Vrba J, Persson M. Evaluation of a patch antenna applicator for time reversal hyperthemia. Int J Hyperthermia. 2010;26(2): 185-197.

3. Lin WL, Shieh MJ, Lui LT, Liauh CT, Wang CY. Ultrasound heating methods for hyperthermic therapy. J Med Ultrason. 1994;2(3): $113-120$

4. Liang A, Liu Q, Wen G, Jiang Z. The surface-plasmon-resonance effect of nanogold/silver and its analytical applications. Trac-Trend Anal Chem. 2012;37:32-47.

5. Huang X, El-Sayed MA. Gold nanoparticles: optical properties and implementations in cancer diagnosis and photothermal therapy. $J$ Avd Res. 2010;1(1):13-28.

6. Panchapakesan B, Book-Newell B, Sethu P, Rao M, Irudayaraj J. Gold nanoprobes for theranostics. Nanomedicine (Lond). 2011;6(10): $1787-1811$

7. Khlebtsov N, Bogatyrev V, Dykman L, et al. Analytical and theranostic applications of gold nanoparticles and multifunctional nanocomposites. Theranostics. 2013;3(3):167-180.

8. Vivero-Escoto JL, Slowing II, Wu CW, Lin VS. Photoinduced intracellular controlled release drug delivery in human cells by goldcapped mesoporous silica nanosphere. J Am Chem Soc. 2009;131(10): 3462-3463.

9. Tsai SW, Liaw JW, Hsu FY, Chen YY, Lyu MJ, Yeh MH. Surfacemodified gold nanoparticles with folic acid as optical probes for cellular imaging. Sensors. 2008;8(10):6660-6673.
10. Qian J, Jiang L, Cai F, Wang D, He S. Fluorescence-surface enhanced Raman scattering co-functionalized gold nanorods as near-infrared probes for purely optical in vivo imaging. Biomaterials. 2011;32(6):1601-1610.

11. Leduc C, Si S, Gautier J, et al. A highly specific gold nanoprobe for livecell single-molecule imaging. Nano Lett. 2013;13(4):1489-1494.

12. Haiss W, Thanh NTK, Aveyard J, Fernig DG. Determination of size and concentration of gold nanoparticles from UV-vis spectra. Anal Chem. 2007;79(11):4215-4221.

13. Khlebtsov NG, Dykman LA. Optical properties and biomedical applications of plasmonic nanoparticles. J Quant Spectrosc Ra. 2010;111(1): $1-35$.

14. Li JL, Gu M. Surface plasmonic gold nanorods for enhanced twophoton microscopic imaging and apoptosis induction of cancer cells. Biomaterials. 2010;31(36):9492-9498.

15. Qu X, Yao C, Wang J, Li Z, Zhang Z. Anti-CD30-targeted gold nanoparticles for photothermal therapy of L-428 Hodgkin's cell. Int J Nanomedicine. 2012;7:6095-6103.

16. Bartczak D, Muskens OL, Millar TM, Sanchez-Elsner T, Kanaras AG. Laser-induced damage and recovery of plasmonically targeted human endothelial cells. Nano Lett. 2011;11(3):1358-1363.

17. Yuan H, Khoury CG, Wilson CM, Grant GA, Bennett AJ, Vo-Dinh T. In vivo particle tracking and photothermal ablation using plasmonresonant gold nanostars. Nanomedicine. 2012;8(8):1355-1363.

18. Melancon MP, Lu W, Yang Z, et al. In vitro and in vivo targeting of hollow gold nanoshells directed at epidermal growth factor receptor for photothermal ablation therapy. Mol Cancer Ther. 2008;7(6):1730-1739.

19. Lalonde BSL, Boulais E, Lebrun JJ, Meunier M. Visible and near infrared resonance plasmonic enhanced nanosecond laser optoporation of cancer cells. Biomed Opt Express. 2013;4(4):490-499.

20. Walkey CD, Olsen JB, Guo H, Emili A, Chan WC. Nanoparticle size and surface chemistry determine serum protein adsorption and macrophage uptake. J Am Chem Soc. 2012;134(4):2139-2147.

21. Chithrani BD, Ghazani AA, Chan WC. Determining the size and shape dependence of gold nanoparticle uptake into mammalian cells. Nano Lett. 2006;6(4):662-668.

22. Lo CL, Chou MH, Lu PL, et al. The effect of PEG-5K grafting level and particle size on tumor accumulation and cellular uptake. Int J Pharm. 2013;456(2):424-431.

23. Yohan D, Cruje C, Lu XF, Chithrani DB. Size-dependent gold nanoparticle interaction at nano-micro interface using both monolayer and multilayer (tissue-like) cell models. Nano-Micro Lett. 2016;8(1):44-53.

24. Zhang X, Dowd DR, Moore MC, et al. Meningioma 1 is required for appropriate osteoblast proliferation, motility, differentiation, and function. J Biol Chem. 2009;284(27):18174-18183.

25. Tsai SW, Liaw JW, Kao YC, et al. Internalized gold nanoparticles do not affect the osteogenesis and apoptosis of MG63 osteoblast-like cells: a quantitative, in vitro study. PLoS One. 2013;8(10):e76545.

26. Liaw JW, Tsai SW, Lin HH, Yen TC, Chen BR. Wavelength-dependent Faraday-Tyndall effect on laser-induced microbubble in gold colloid. J Quant Spectrosc Ra. 2012;113(17):2234-2242.

27. Gregory CA, Gunn WG, Peister A, Prockop DJ. An Alizarin red-based assay of mineralization by adherent cells in culture: comparison with cetylpyridinium chloride extraction. Anal Biochem. 2004;329(1): 77-84.

28. Raji V, Kumar J, Rejiya CS, Vibin M, Shenoi VN, Abraham A. Selective photothermal efficiency of citrate capped gold nanoparticles for destruction of cancer cells. Exp Cell Res. 2011;317(14):2052-2058.

29. Krpetić Z, Nativo P, Sée V, Prior IA, Brust M, Volk M. Inflicting controlled nonthermal damage to subcellular structures by laser-activated gold nanoparticles. Nano Lett. 2010;10(11):4549-4554.

30. Krawinkel J, Richter U, Torres-Mapa ML, et al. Optical and electron microscopy study of laser-based intracellular molecule delivery using peptide-conjugated photodispersible gold nanoparticle agglomerates. J Nanobiotechnology. 2016;14(1):2.

31. Metwally K, Mensah S, Baffou G. Fluence threshold for photothermal bubble generation using plasmonic nanoparticles. J Phys Chem C. 2015; 119(51):28586-28596. 
32. Lapotko D. Plasmonic nanoparticle-generated photothermal bubbles and their biomedical applications. Nanomedicine (Lond). 2009;4(7) 813-845.

33. Mvula B, Moore TJ, Abrahamse H. Effect of low-level laser irradiation and epidermal growth factor on adult human adipose-derived stem cells. Lasers Med Sci. 2010;25(1):33-39.

34. Soleimani M, Abbasnia E, Fathi M, Sahraei H, Fathi Y, Kaka G. The effects of low-level laser irradiation on differentiation and proliferation of human bone marrow mesenchymal stem cells into neurons and osteoblasts-an in vitro study. Lasers Med Sci. 2012;27(2):423-430.
35. Yi C, Liu D, Fong CC, Zhang J, Yang M. Gold nanoparticles promote osteogenic differentiation of mesenchymal stem cells through p38 MAPK pathway. ACS Nano. 2010;4(11):6439-6448.

36. Sathuluri RR, Yoshikawa H, Shimizu E, Saito M, Tamiya E. Gold nanoparticle-based surface-enhanced Raman scattering for noninvasive molecular probing of embryonic stem cell differentiation. PLoS One. 2011;6(8):e22802. 


\section{Supplementary materials}
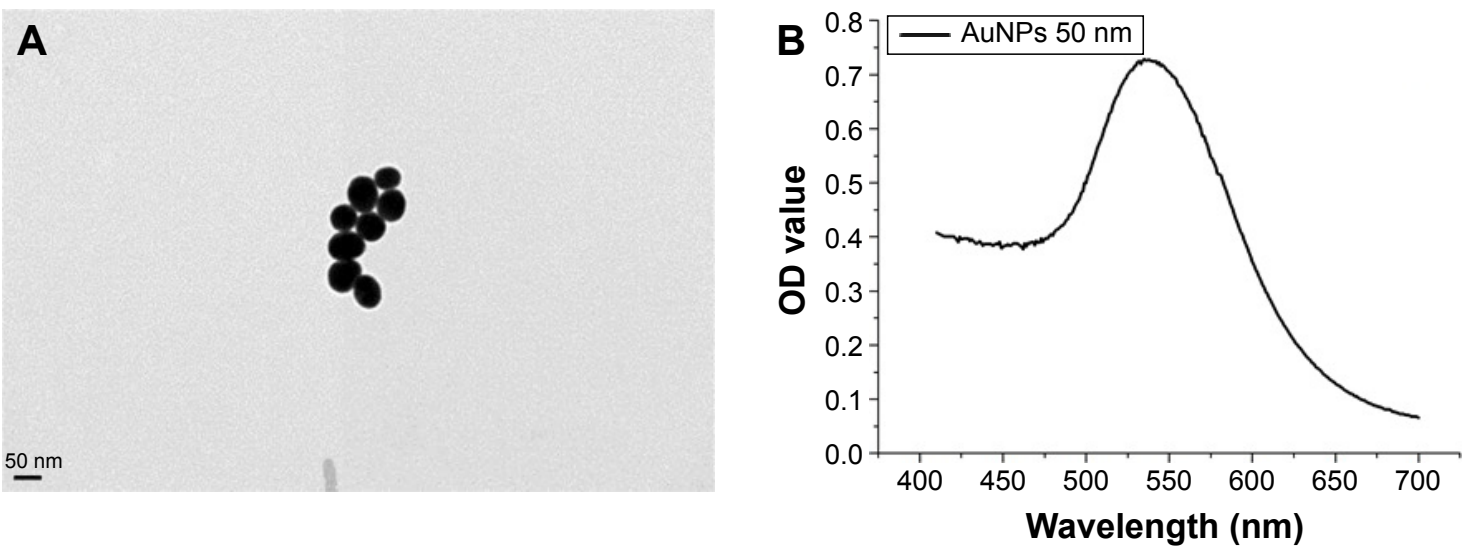

Figure SI TEM image of AuNPs (A) and UV-vis adsorption spectrum of AuNPs (B).

Abbreviations: TEM, transmission electron microscopic; AuNP, gold nanoparticle; UV-vis, ultraviolet-visible; OD, optical density.
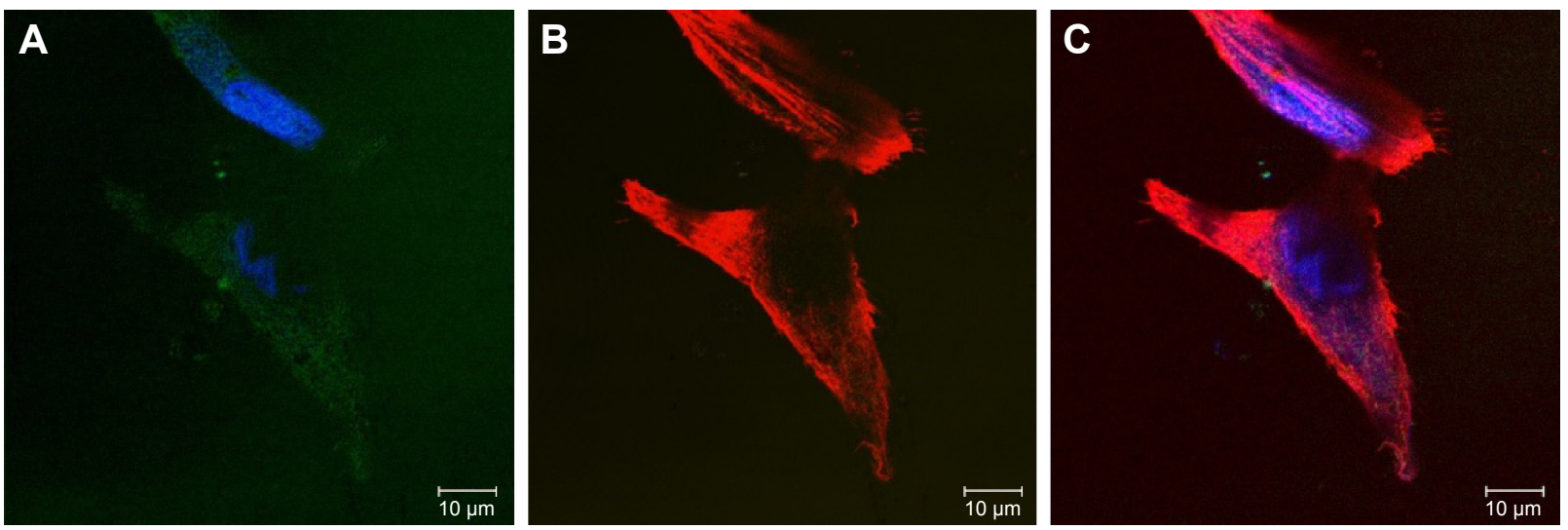

Figure S2 Confocal microscopy photograph of cells treated with $\mathrm{H}_{2} \mathrm{O}_{2}$.

Notes: (A) Annexin V-Alexa Fluor 488 was used to indicate PS, shown in green; DAPI was used to stain nuclei, shown in blue; (B)Texas Red-X phalloidin was used to label cytoskeletal F-actin, shown in red; and $(\mathbf{C})$ superimposition of $(\mathbf{A})$ and $(\mathbf{B})$.

Abbreviations: PS, phosphatidylserine; DAPI, 4',6-diamidino-2-phenylindole.

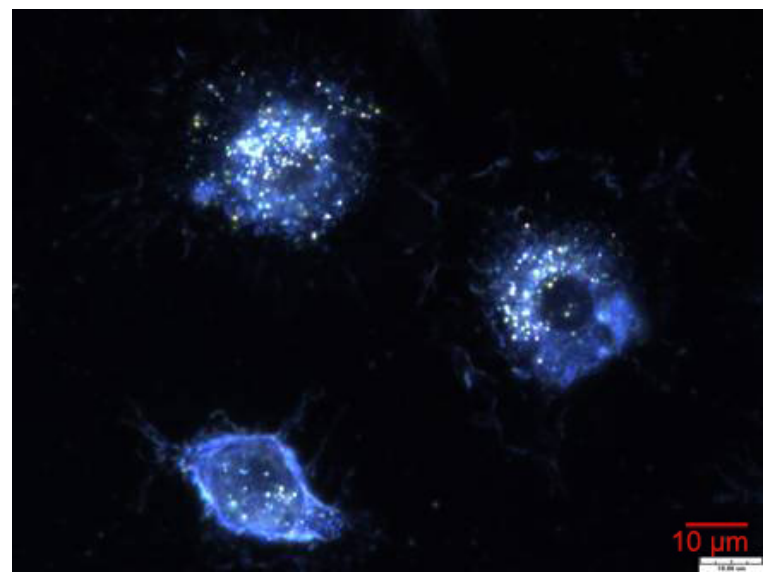

Figure S3 Dark-field image of cells after treated with AuNPs and laser irradiation of $100 \mathrm{~mW} / \mathrm{cm}^{2}$.

Abbreviation: AuNP, gold nanoparticle. 
International Journal of Nanomedicine

Dovepress

\section{Publish your work in this journal}

The International Journal of Nanomedicine is an international, peerreviewed journal focusing on the application of nanotechnology in diagnostics, therapeutics, and drug delivery systems throughout the biomedical field. This journal is indexed on PubMed Central, MedLine, CAS, SciSearch ${ }^{\circledR}$, Current Contents ${ }^{\circledR} /$ Clinical Medicine,
Journal Citation Reports/Science Edition, EMBase, Scopus and the Elsevier Bibliographic databases. The manuscript management system is completely online and includes a very quick and fair peer-review system, which is all easy to use. Visit http://www.dovepress.com/ testimonials.php to read real quotes from published authors.

Submit your manuscript here: http://www.dovepress.com/international-journal-of-nanomedicine-journal 